\title{
Gallstone pancreatitis: general clinical approach and the role of endoscopic retrograde cholangiopancreatography
}

\author{
Shanker Kundumadam, Evan L. Fogel, and Mark Andrew Gromski
}

Division of Gastroenterology and Hepatology, Indiana University School of Medicine, Indianapolis, IN, USA

\section{Received: October 2, 2020}

Accepted: November 2, 2020

\section{Correspondence to}

Mark Andrew Gromski, M.D. Division of Gastroenterology and Hepatology, Indiana University School of Medicine, 550 N. University Blvd, Suite 1634, Indianapolis, IN 46202, USA

Tel: +1-317-944-0925

Fax: +1-317-968-1265

E-mail: mgromski@iu.edu https://orcid.org/0000-00029035-2434
Gallstones account for majority of acute pancreatitis in the Western world. Increase in number and smaller size of the stones increases the risk for biliary pancreatitis. In addition to features of acute pancreatitis, these patients also have cholestatic clinical picture. Fluid therapy and enteral nutrition are vital components in management of any case of acute pancreatitis. During initial evaluation, a right upper quadrant ultrasonogram is particularly important. On a case-bycase basis, further advanced imaging studies such as magnetic resonance cholangiopancreatography or endoscopic ultrasound may be warranted. Acute management also involves monitoring for local and systemic complications. Patients are triaged based on predictors of ongoing biliary obstruction in order to identify who would need endoscopic retrograde cholangiopancreatography. Index cholecystectomy is safe and recommended, with exception of cases with significant local and systemic complications where delayed cholecystectomy may be safer.

Keywords: Pancreatitis; Cholangiopancreatography, endoscopic retrograde; Cholecystectomy

\section{INTRODUCTION}

Acute pancreatitis is a condition characterized by acute inflammation of the pancreas [1]. There are multiple risk factors and putative etiologies of acute pancreatitis; however, gallstones and alcohol are the identified etiology in up to $70 \%$ of cases $[2,3]$. In the Western world, gallstones are the leading cause of acute pancreatitis, causing up to $40 \%$ of cases [4]. For patients who harbor gallstones, the risk of biliary pancreatitis is up to $7 \%$ on long term follow-up [5]. Multiple hypotheses exist to explain the mechanism by which stones cause pancreatitis. Invariably, the stone must exit the gallbladder through the cystic duct and cause acute pancreatitis from being lodged at the pancreatobiliary junction or alternatively, a primary bile duct stone or secondary gallbladder stone may pass through the ampulla of Vater. Ampullary obstruction, local inflammation due to stone passage at the level of the ampulla of Vater and transient bile reflux into the pancreatic duct are all potential mechanisms to explain gallstone pancreatitis [6]. The risk of gallstone pancreatitis increases with smaller gallstones (allowing more unrestricted passage into the common bile duct and across the ampulla), and increased number of gallstones [7].

Acute pancreatitis of any etiology is diagnosed if two of the following three criteria are filled:(1) upper abdominal or back pain consistent with pancreas type pain, (2) increased pancreatic enzyme levels (lipase or amylase) > 3 times the upper limit of normal, and (3) pancreatic in- 


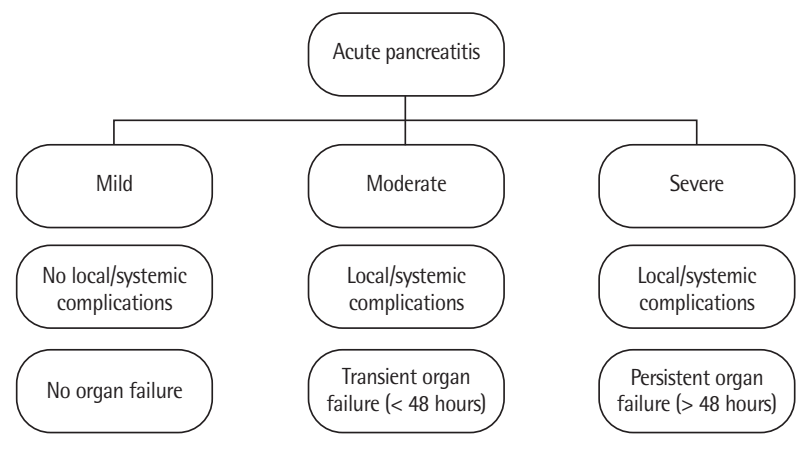

Figure 1. Revised Atlanta classification on severity of pancreatitis.

flammation on abdominal imaging (usually computed tomography [CT] or magnetic resonance imaging) $[8,9]$. The abdominal pain is usually epigastric in location and radiates to the thoracic back, but may also be solely located in the back or in the left or right upper quadrants. Nausea and emesis are also often predominant symptoms [10]. The revised Atlanta classification is widely used to stratify the severity of acute pancreatitis, based on the presence or absence of local/systemic complications and the presence or absence of concomitant organ failure (Fig. 1) [9]. Due to this wide range in presentation, physical examination findings can vary significantly from patient to patient. In addition to the classically described clinical findings of abdominal tenderness, other clinical signs of ileus (distended, tympanic abdomen) and features of systemic inflammatory response syndrome (SIRS) may be encountered. Characteristics of biliary obstruction including dark urine, pale stools, icteric sclerae, excoriations from pruritis and jaundice may be valuable clues pointing towards gallstones as the etiological culprit for an episode of acute pancreatitis. The presence of risk factors for gallstones, such as older age, female gender, obesity, rapid weight loss, pregnancy or hormone therapy with estrogen may also increase the level of suspicion for biliary pancreatitis [11]. Elevated liver tests at the time of presentation for acute pancreatitis also raises this suspicion. Specifically, an elevated alanine transaminase level more than three times upper limit of normal (with a greater level than the aspartate aminotransferase) is a relatively specific indication of a biliary etiology under appropriate circumstances [12].

\section{GENERAL APPROACH TO MANAGEMENT}

\section{Fluid therapy and nutrition}

Patients suspected to have acute pancreatitis or diagnosed with acute pancreatitis should be appropriately triaged and resuscitated. The American College of Gastroenterology guidelines for the management of acute pancreatitis published in 2013 recommend admission to the intensive care unit for patients who present with organ failure or fulfilling SIRS criteria [8]. Fluid therapy remains of utmost importance in management of acute pancreatitis. Judicious goal-directed intravenous (IV) fluid therapy with normal saline or Ringer's lactate has been recommended by the American Gastroenterological Association (AGA) in its guideline published in 2018. The titration of fluids to clinical and biochemical targets of perfusion such as blood urea nitrogen, hematocrit, heart rate and mean arterial pressure is appropriate [13]. After an initial bolus of IV fluids, we often start IV hydration with Ringer's lactate at a rate of $3 \mathrm{cc} / \mathrm{kg} / \mathrm{hr}$ for the first 24 hours in adults, with some variability based on ideal body weight, co-morbidities (for instance renal, cardiac or pulmonary disease) and clinical scenario. Overhydration should be avoided as this may increase the risk of acute respiratory distress syndrome (ARDS) $[14,15]$. It is also important to initiate early oral feeding within 24 hours. Enteral feeding with a nasogastric or nasojejunal tube is appropriate in patients unable to tolerate oral feeding. Prophylactic antibiotics are not recommended in routine cases of acute pancreatitis where infection is not suspected [13].

\section{Imaging studies}

An ultrasound (US) of the abdomen is the most appropriate imaging study in the initial evaluation of suspected gallstone pancreatitis. It has a sensitivity and specificity of $84 \%$ and $99 \%$, respectively, in identifying gallbladder stones [16]. This also facilitates visualization of the biliary tract to identify bile duct stones or biliary dilatation, which increases the suspicion for biliary obstruction. The integration of clinical findings (presence or absence of jaundice), US findings (presence or absence of gallstones in gallbladder, stones in common bile duct or biliary dilation), along with laboratory test results (transaminases, alkaline phosphatase, and bili- 
rubin levels) facilitate decision-making on predicting the probability of persistent biliary obstruction and the need for endoscopic intervention in cases of suspected gallstone pancreatitis. If a patient meets the diagnosis for acute pancreatitis without cross-sectional imaging (having abdominal pain and pancreas enzymes $>3$ times

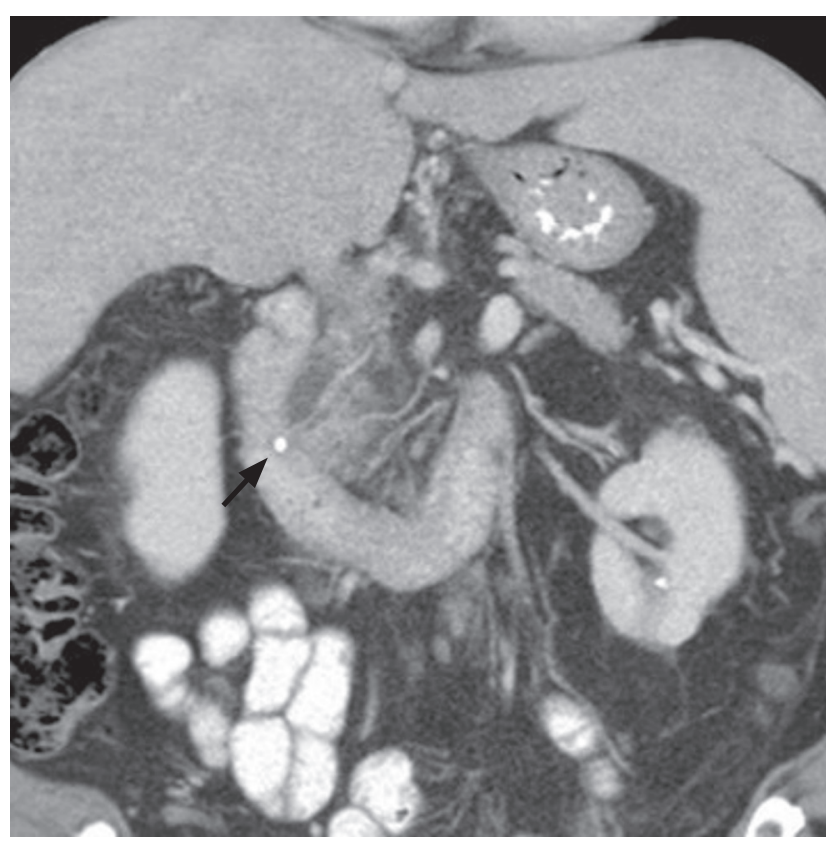

Figure 2. Coronal series computed tomography scan image showing an impacted stone in the terminal bile duct (arrow), with relative proximal dilation of the common bile duct and peripancreatic edema and stranding in the head of the pancreas. the upper limit of normal), a CT scan is not necessary on initial evaluation. A CT scan may, at times, identify an impacted stone at the level of the ampulla of Vater (Fig. 2); however, this is not the most sensitive radiographic test for detection of biliary stones. If there continues to be a clinical question after initial work-up for the etiology of acute pancreatitis, magnetic resonance cholangiopancreatography (MRCP) or endoscopic ultrasound (EUS) (Fig. 3) have better performance characteristics for detecting common bile duct stones than CT scan [17].

\section{Monitoring for complications}

During the management of acute pancreatitis, potential local and systemic complications should be anticipated. Local complications include acute peripancreatic fluid collections, acute necrotic collections indicative of pancreas necrosis, mesenteric vein thrombosis, and infection. Deteriorating clinical status, persistent abdominal pain, ongoing fever, or persistent inability to tolerate oral intake should prompt the clinician to suspect local complications and consider new cross-sectional imaging (CT with IV contrast, as appropriate). Systemic complications include SIRS, ARDS, and presence of other single or multiple organ failure $[9,18]$.

\section{Pre-procedural optimization}

In the setting of gallstone pancreatitis, invasive procedures such as EUS, endoscopic retrograde cholangiopancreatography (ERCP) or cholecystectomy may be
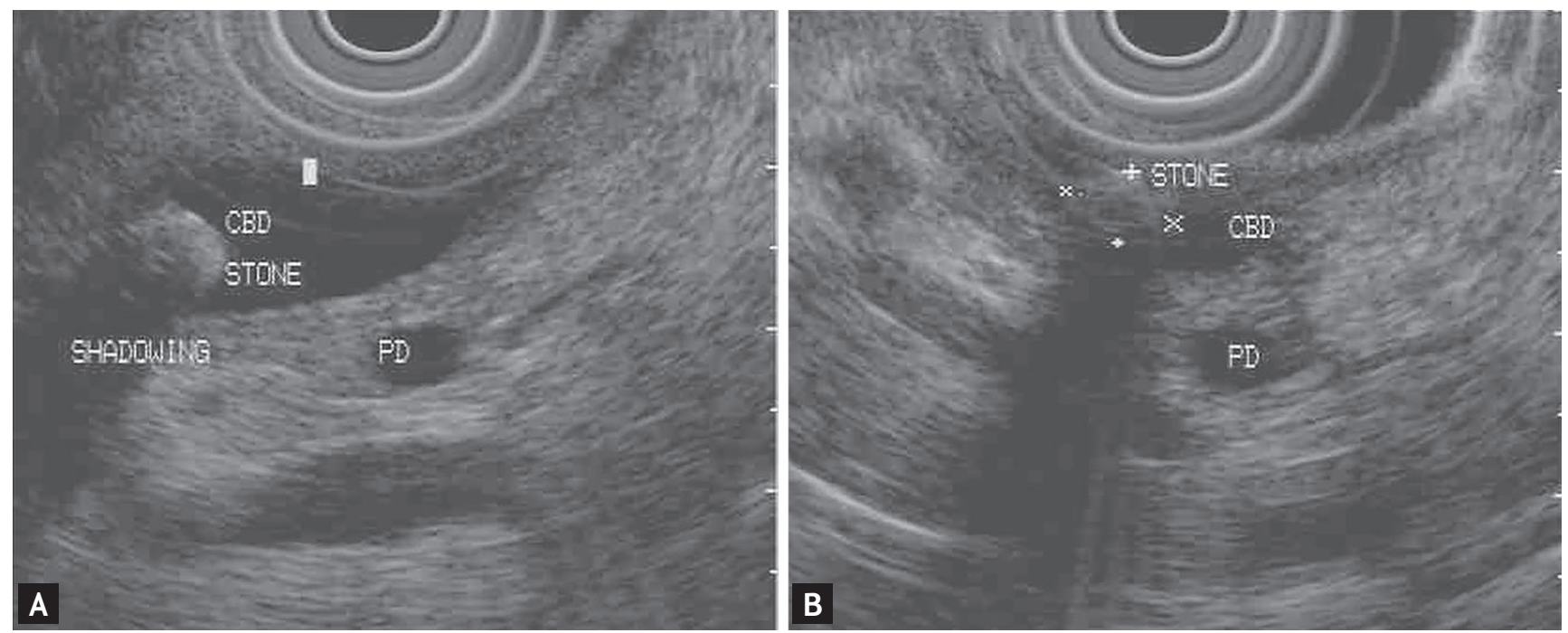

Figure 3. Endoscopic ultrasound images of (A) a biliary stone demonstrating classic hyperechogenicity and distal shadowing and (B) terminal bile duct stone near the ampulla. CBD, common bile duct; PD, pancreatic duct. 


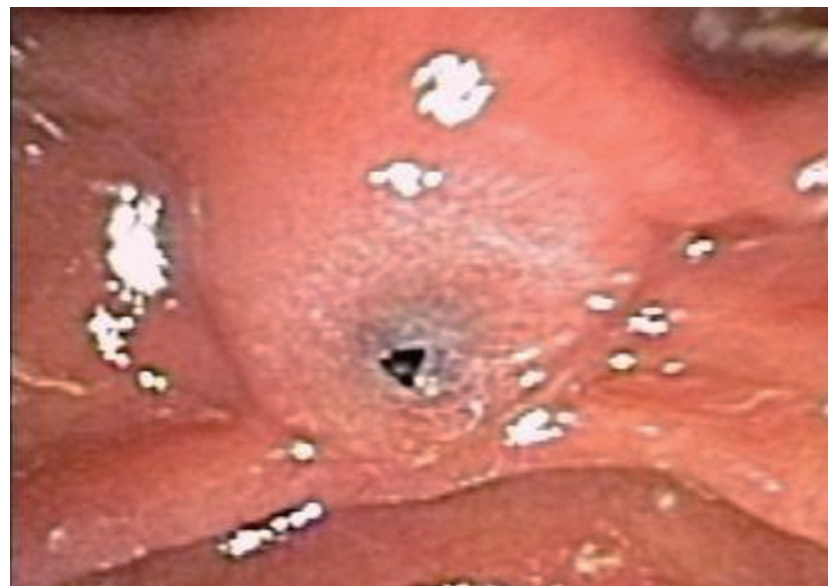

Figure 4. Pigmented bile duct stone present at the biliary orifice of the major papilla.

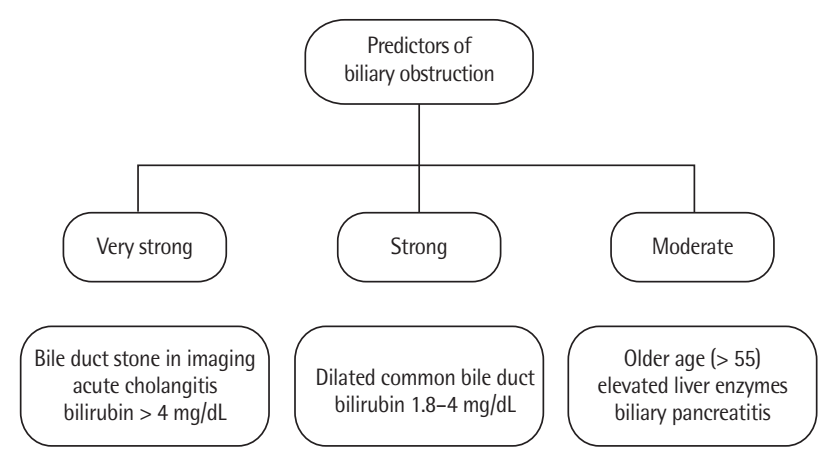

Figure 6. Factors predicting biliary obstruction requiring intervention in the setting of acute pancreatitis.

indicated during the index hospitalization. Cholestasis and associated malabsorption of vitamin $\mathrm{K}$ can impair coagulation in patients with gallstone pancreatitis, which may increase the risk for procedural and post procedural bleeding. In cases where surgery or endoscopy is anticipated, coagulation parameters including prothrombin time and international normalized ratio should be monitored and corrected. Ensuring intravascular volume repletion and provision of antibiotics in cases of suspected ascending cholangitis are also crucial in the optimization of patients for an invasive procedure $[12,19]$.

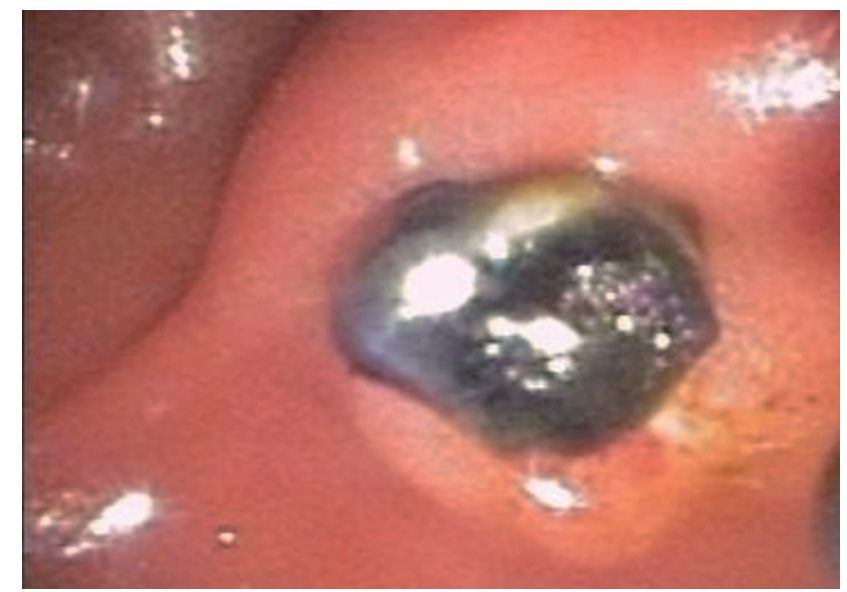

Figure 5. Removal of pigmented bile duct stone after endoscopic sphincterotomy.

\section{ROLE OF ERCP}

ERCP is an endoscopic procedure that uses a side viewing duodenoscope which facilitates identification of the ampulla of Vater and cannulation of the biliary or pancreatic ductal system. Once cannulated, contrast is injected and, with fluoroscopic assistance, the biliary or pancreatic ducts may be visualized [20-22]. If there is suspicion or confirmation of cholangitis, ERCP allows for the aspiration of bile for culture and sensitivity studies, along with drainage of the biliary system by either removing bile duct stones or placing a biliary stent [23]. For management of choledocholithiasis, a sphincterotomy is performed, which involves cutting the biliary sphincter with an electrosurgical sphincterotome. Retrieval of the stones (Figs. 4 and 5) at ERCP is typically done using a balloon or basket, but larger stones may require further maneuvers such as endoscopic fragmentation with lithotripsy. Complete removal of the stones may not be feasible during an index ERCP (particularly if there is concern for ascending cholangitis), and these instances warrant a biliary stent to be placed to ensure drainage [12]. Advanced stone extraction maneuvers may be performed at a subsequent procedure.

Whether or not to pursue ERCP in the setting of gallstone pancreatitis is an important clinical decision. Routine performance of ERCP for all patients with gallstone pancreatitis is not recommended, except in certain situations [13]. Fig. 6 illustrates those factors that al- 
ter the likelihood of ongoing biliary obstruction, which may impact the decision to proceed with ERCP. If a very strong predictor for ongoing biliary obstruction is present, such as ascending cholangitis, choledocholithiasis seen on an imaging study or total bilirubin $>4 \mathrm{mg} / \mathrm{dL}$, then ERCP is indicated [12,13,17]. If a strong predictor is present without the factors above (biliary dilation on imaging study or elevated bilirubin 1.8 to $4 \mathrm{mg} / \mathrm{dL}$ ), then an adjunct imaging test for choledocholithiasis (EUS or MRCP) is indicated. In patients who do not satisfy the above criteria but there remains suspicion for ongoing biliary obstruction, a high-quality evaluation of the biliary system with MRCP or EUS is warranted. If that evaluation shows evidence of a common bile duct stone, then proceeding with ERCP is the preferred course. Aside from the above situations of ascending cholangitis or persistent biliary obstruction, ERCP is unlikely to change the course of the disease, nor prevent any local or systemic complications or mortality of acute pancreatitis [24] and is discouraged [25].

A recent study by Muangkaew et al. [26] as well as the Tokyo guidelines have noted that in cases of biliary pancreatitis with mild cholangitis, conservative medical management and delaying ERCP may not impact morbidity or mortality [27]. Nevertheless, if ERCP is readily available, we recommend prompt (within 48 hours of presentation) ERCP for all patients with gallstone pancreatitis and suspicion of ascending cholangitis.

\section{ROLE OF CHOLECYSTECTOMY}

The AGA recommends laparoscopic cholecystectomy during the index admission for gallstone pancreatitis, once the pancreatitis has resolved [28]. Cholecystectomy performed at this time has been shown to decrease the risk of recurrent biliary events (cholangitis, symptomatic cholelithiasis or choledocholithiasis, recurrent pancreatitis) and to decrease readmissions [29-31]. This is, however, not always feasible or prudent, especially in cases of moderate or severe pancreatitis. It is reasonable in scenarios of severe acute pancreatitis, pancreas necrosis or significant peripancreatic fluid collections to opt for a delayed cholecystectomy rather than one performed on index presentation. This approach is associated with lesser morbidity and mortality in these unique scenarios [32]. In certain circumstances a cholecystectomy may not be feasible or may be contraindicated. Examples include advanced age, decompensated cirrhosis or coagulopathy. An ERCP with biliary sphincterotomy or transpapillary biliary or gallbladder stent in these instances may prevent recurrence of biliary pancreatitis $[12,33,34]$.

\section{CONCLUSIONS}

Gallstones are the most encountered cause of acute pancreatitis. Early management with appropriate triage and fluid resuscitation with detection of local or systemic complications is of paramount importance. In gallstone pancreatitis, the decision on the need and timing of procedural intervention is crucial. ERCP is indicated for ascending cholangitis or clear evidence of ongoing biliary obstruction in this setting. Finally, cholecystectomy is indicated during the index admission for most cases of acute gallstone pancreatitis.

\section{Conflict of interest}

No potential conflict of interest relevant to this article was reported.

\section{REFERENCES}

1. Vege SS. Acute pancreatitis. In: Feldman M, Friedman L, Brandt L, ed. Sleisenger and Fordtran's Gastrointestinal and Liver Disease. 1oth ed. Philadelphia (PA): Elsevier/ Saunders, 2016:969-993.

2. Attasaranya S, Fogel EL, Lehman GA. Choledocholithiasis, ascending cholangitis, and gallstone pancreatitis. Med Clin North Am 2008;92:925-960.

3. Fogel EL, Sherman S. Diseases of the gallbladder and bile ducts. In: Goldman L, Schafer A, ed. Goldman-Cecil Medicine. 26th ed. Philadelphia (PA): Elsevier, 2020;10051015 .

4. Yadav D, Lowenfels AB. Trends in the epidemiology of the first attack of acute pancreatitis: a systematic review. Pancreas 2006;33:323-330.

5. Moreau JA, Zinsmeister AR, Melton LJ 3rd, DiMagno EP. Gallstone pancreatitis and the effect of cholecystectomy: a population-based cohort study. Mayo Clin Proc 
1988;63:466-473.

6. Lerch MM, Saluja AK, Runzi M, Dawra R, Saluja M, Steer ML. Pancreatic duct obstruction triggers acute necrotizing pancreatitis in the opossum. Gastroenterology 1993;104:853-861.

7. Venneman NG, Renooij W, Rehfeld JF, et al. Small gallstones, preserved gallbladder motility, and fast crystallization are associated with pancreatitis. Hepatology 2005;41:738-746.

8. Tenner S, Baillie J, DeWitt J, Vege SS; American College of Gastroenterology. American College of Gastroenterology guideline: management of acute pancreatitis. Am J Gastroenterol 2013;108:1400-1415.

9. Banks PA, Bollen TL, Dervenis C, et al. Classification of acute pancreatitis: 2012. Revision of the Atlanta classification and definitions by international consensus. Gut 2013;62:102-111.

10. Banks PA, Freeman ML; Practice Parameters Committee of the American College of Gastroenterology. Practice guidelines in acute pancreatitis. Am J Gastroenterol 2006;101:2379-2400.

11. Stinton LM, Shaffer EA. Epidemiology of gallbladder disease: cholelithiasis and cancer. Gut Liver 2012;6:172-187.

12. Fogel EL, Sherman S. ERCP for gallstone pancreatitis. N Engl J Med 2014;370:150-157.

13. Crockett SD, Wani S, Gardner TB, Falck-Ytter Y, Barkun AN; American Gastroenterological Association Institute Clinical Guidelines Committee. American Gastroenterological Association Institute guideline on initial management of acute pancreatitis. Gastroenterology 2018;154:1096-1101.

14. de-Madaria E, Soler-Sala G, Sanchez-Paya J, et al. Influence of fluid therapy on the prognosis of acute pancreatitis: a prospective cohort study. Am J Gastroenterol 2011;106:1843-1850.

15. Tess A, Freedman SD, Kent T, Libman H. How would you treat this patient with gallstone pancreatitis?: grand rounds discussion from Beth Israel Deaconess Medical Center. Ann Intern Med 2019;170:175-181.

16. Shea JA, Berlin JA, Escarce JJ, et al. Revised estimates of diagnostic test sensitivity and specificity in suspected biliary tract disease. Arch Intern Med 1994;154:2573-2581.

17. Easler JJ, Sherman S. Endoscopic retrograde cholangiopancreatography for the management of common bile duct stones and gallstone pancreatitis. Gastrointest Endosc Clin N Am 2015;25:657-675.
18. Zerem E. Treatment of severe acute pancreatitis and its complications. World J Gastroenterol 2014;20:13879-13892.

19. Papadopoulos V, Filippou D, Manolis E, Mimidis K. Haemostasis impairment in patients with obstructive jaundice. J Gastrointestin Liver Dis 2007;16:177-186.

20. Kim J. Training in endoscopy: endoscopic retrograde cholangiopancreatography. Clin Endosc 2017;50:334-339.

21. Cotton PB. Cannulation of the papilla of Vater by endoscopy and retrograde cholangiopancreatography (ERCP). Gut 1972;13:1014-1025.

22. Cotton PB, Leung J. Advanced Digestive Endoscopy: ERCP. Oxford (UK): Blackwell Publishing Ltd., 2007.

23. Rerknimitr R, Fogel EL, Kalayci C, Esber E, Lehman GA, Sherman S. Microbiology of bile in patients with cholangitis or cholestasis with and without plastic biliary endoprosthesis. Gastrointest Endosc 2002;56:885-889.

24. Tse F, Yuan Y. Early routine endoscopic retrograde cholangiopancreatography strategy versus early conservative management strategy in acute gallstone pancreatitis. Cochrane Database Syst Rev 2012;(5):CDoo9779.

25. ASGE Standards of Practice Committee, Buxbaum JL, Abbas Fehmi SM, et al. ASGE guideline on the role of endoscopy in the evaluation and management of choledocholithiasis. Gastrointest Endosc 2019;89:1075-1105.

26. Muangkaew P, Kamalaporn P, Mingphruedhi S, et al. Outcomes of delayed endoscopic retrograde cholangiopancreatography in patients with acute biliary pancreatitis with cholangitis. Asian J Surg 2020;43:913-918.

27. Miura F, Okamoto K, Takada T, et al. Tokyo guidelines 2018: initial management of acute biliary infection and flowchart for acute cholangitis. J Hepatobiliary Pancreat Sci 2018;25:31-40.

28. Vege SS, DiMagno MJ, Forsmark CE, Martel M, Barkun AN. Initial medical treatment of acute pancreatitis: American Gastroenterological Association Institute technical review. Gastroenterology 2018;154:1103-1139.

29. Moody N, Adiamah A, Yanni F, Gomez D. Meta-analysis of randomized clinical trials of early versus delayed cholecystectomy for mild gallstone pancreatitis. Br J Surg 2019;106:1442-1451.

30. Krishna SG, Kruger AJ, Patel N, Hinton A, Yadav D, Conwell DL. Cholecystectomy during index admission for acute biliary pancreatitis lowers 30-day readmission rates. Pancreas 2018;47:996-1002.

31. van Baal MC, Besselink MG, Bakker OJ, et al. Timing of cholecystectomy after mild biliary pancreatitis: a system- 
atic review. Ann Surg 2012;255:86o-866.

32. Nealon WH, Bawduniak J, Walser EM. Appropriate timing of cholecystectomy in patients who present with moderate to severe gallstone-associated acute pancreatitis with peripancreatic fluid collections. Ann Surg 2004;239:741749 .

33. Swisher SG, Hunt KK, Schmit PJ, Hiyama DT, Bennion
RS, Thompson JE. Management of pancreatitis complicating pregnancy. Am Surg 1994;60:759-762.

34. Garcia de la Filia Molina I, Garcia Garcia de Paredes A, Martinez Ortega A, et al. Biliary sphincterotomy reduces the risk of acute gallstone pancreatitis recurrence in non-candidates for cholecystectomy. Dig Liver Dis 2019;51:1567-1573. 